Trauma Berufskrankh 2006 • 8[Suppl 3]:S329-S332 DOI 10.1007/s10039-005-1066-y

Online publiziert: 8. Dezember 2005

๑) Springer Medizin Verlag 2005

H.-E. Schaller $\cdot$ N. Sinis

Klinik für Plastische, Hand-, Rekonstruktive und Verbrennungschirurgie, BG-Unfallklinik, Eberhard-Karls-Universität Tübingen

\title{
Behandlung des Morbus Sudeck
}

\section{CRPS-Symptomatik}

Leitsymptome. Oft zeichnet sich ein stadienhafter Verlauf ab, wobei als Leitsymptom immer der Schmerz im Vordergrund steht [20]. Besonders störend wird von den Patienten die Allodynie empfunden. Hier kommt es bereits bei kleinster Berührung zu einer überschießenden Schmerzwahrnehmung.

Zum klinischen Bild gehören auch die autonomen Störungen, wodurch Hautveränderungen mit Effloreszenzen sowie Hyper- oder Hypopigmentierungen möglich sind. Außerdem können Hypertrichose, Ödembildung und Glanzhaut auftreten. Besonders die teigige Schwellung imponiert häufig zu Beginn der Erkrankung [3]. Weiterhin wurden gestörtes Wachstum der Fingernägel sowie in schweren Fällen Durchblutungsstörungen und Ulzerationen beobachtet [29].

Diese autonomen Veränderungen werden unter dem Begriff der trophischen Störungen zusammengefasst. Temperaturunterschiede zur Gegenseite kommen ebenfalls vor und dokumentieren eine veränderte Durchblutungssituation bzw. eine Entgleisung der Regulationsmechanismen, die für diese verantwortlich sind.

Generalisierung. Sowohl durch die starken Schmerzen als auch aufgrund der Schwellungszustände kommt es zu massiven Bewegungseinschränkungen, die sich auch in Form eines Intentionstremors, als zentraler Komponente, widerspiegeln können [18]. In der Regel breitet sich die Symptomatik über das traumatisierte Areal hinaus aus. In seltenen Fällen ist die gesamte Körperhälfte betroffen [12].
Aufgrund des chronischen Verlaufs wird ein Großteil der Patienten, abhängig von ihrer psychosozialen Situation, verhaltensauffällig, sodass therapeutisch eine psychologische Mitbetreuung ratsam ist [5].

\section{Pathophysiologie}

Bezüglich der genauen CRPS-Entstehungsmechanismen besteht weiterhin Unklarheit [2]. Als auslösendes Trauma genügt eine Bagatellverletzung. Das Ausmaß der Symptomatik korreliert nur selten mit dem später geäußerten Beschwerdebild. Aufgrund tierexperimenteller Studien wird eine überproportionale Stimulation von nozizeptiven Afferenzen angenommen [11]. Weiterhin konnte in einem Rattenmodell, in dem artifiziell ein CRPS induziert wurde, eine Zunahme von NMDA-Rezeptoren im ZNS nachgewiesen werden [28]. Mittlerweile ist das Vorhandensein von zentralen Veränderungen, insbesondere auf kortikaler Ebene, aufgrund zahlreicher in der Vergangenheit publizierter Arbeiten als pathophysiologischer Faktor allgemein anerkannt [4].

Wir konnten in eigenen Studien nachweisen, dass bei Blockade der NMDA-Rezeptoren eine signifikante Remission der kortikalen Veränderungen stattfindet. Weiterhin kam es zu einer deutlichen Verbesserung des klinischen Gesamtbildes hinsichtlich trophischer Störungen, Bewegungsumfänge und Schmerzangabe der Patienten [22].

Die zentralen Veränderungen können jedoch die peripheren Symptome nicht hinreichend erklären, insbesondere auch die teigige Schwellung, die als Folge auch lokaler Veränderungen auf- 


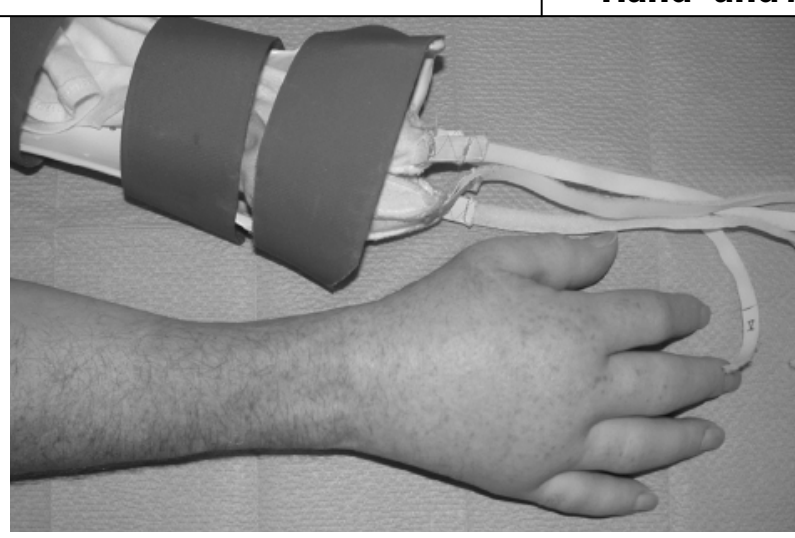

Abb. $1<$ Ödembildung, Hypertrichose, Rötung und prominente Schwellung bei einem 42-jährigen Patienten mit fulminanter CRPS-Form nach Handgelenkdistorsion

tritt. Aus histologischen Präparaten aus dem Gewebe von CRPS-Patienten wurde eine veränderte Rezeptordichte der Katecholaminrezeptoren nachgewiesen [7]. Hierdurch kommt es zu Perfusionsveränderungen im Gewebe, die zu einer Erhöhung des kapillaren Filtrationsdrucks führen. Daraus resultieren Ödembildung und Schwellung in der betroffenen Extremität, wie sie häufig im akuten Stadium als klinisches Bild eindrucksvoll imponieren (• Abb. 1) [23].

Sowohl durch die Immobilisation (iatrogen oder schmerzbedingt) als auch durch die Ödembildung und Stoffwechsellage kommt es an den Bandapparaten und Gelenkkapseln zu vermehrter Narbenbildung und den allseits bekannten schweren Kontrakturen der Fingergelenke.

\section{Diagnose}

Sie wird in der Regel aufgrund des klinischen Bildes und nach Ausschluss anderer Differenzialdiagnosen gestellt [28]. Hierbei ist besonders an rheumatische Erkrankungen, Arthrosen oder Nervenkompressionssyndrome zu denken.

Klinisch ist eine Röntgenaufnahme der betroffenen Hand in einer vergleichenden Aufnahme mit der gesunden Gegenseite häufig richtungweisend, da es zu lokalen Entkalkungen und fleckförmigen Demineralisationszeichen kommt. Im ersten Jahr nach Entstehung eines CRPS ist auch die 3-Phasen-Skelett-Szintigraphie häufig positiv, sodass sie zum Ausschluss zweifelhafter Verläufe (Aggravationsverhalten, usw.) und zur Diagnosestellung herangezogen werden kann [30]. Verschiedene thermographische Verfahren sollen Temperaturunterschiede zur gesunden Gegenseite aufdecken, wobei deren klinische Relevanz eher gering einzuschätzen ist, zumal das klinische Bild in der Regel im Tastbefund sehr deutlich ausfällt. Zur Verifizierung einer gestörten Funktion der Hautanhangsgebilde (Schweißdrüsenfunktion) wird der Ninhydrintest empfohlen, um Unterschiede in der Hautbefeuchtung zu erfassen. Zum Ausschluss von peripheren Nervenverletzungen, die bei einem CRPS Typ II auftreten, wird neben der eingehenden klinischen Untersuchung (2-Punkte-Deskrimination, Sensibilitätsprüfung, Kraftgrade, usw.) auch eine elektrophysiologische Diagnostik empfohlen [28].

\section{Therapie}

\section{Grundsätze/interdisziplinärer Ansatz}

Zunächst steht eine symptomorientierte Behandlungsstrategie im Vordergrund, deren vordringliches Ziel die Behandlung der Schmerzen, Bewegungseinschränkungen sowie der trophischen Veränderungen ist. Bezüglich einer suffizienten Schmerzmedikation sollte stets die Zusammenarbeit mit einem Schmerztherapeuten gesucht werden [26]. Ein interdisziplinäres Vorgehen ist immer wünschenswert und berücksichtigt die unterschiedlichen $\mathrm{Be}$ handlungsdisziplinen ( $\bullet$ Tabelle 1 ).

Die Therapie wird mit den üblichen Basisanalgetika begonnen (nichtsteroidale Antirheumatika usw.). Aufgrund des Feedbacks durch den Patienten kann die Analgetikagabe gesteigert oder mit Medikamenten aus anderen Gruppen kombiniert werden (Opioide, Antidepressiva, $\beta$-Blocker usw.) [21].

Aufgrund des bereits oben erwähnten positiven Effekts eines Psychologen auf die Wahrnehmung und Verarbeitung chronischer Schmerzen sollte dieser in die Behandlungsstrategie integriert werden.
Hierbei kommen die Verhaltens- und Gesprächstherapie zur Anwendung, wobei die Zugänglichkeit für diese Behandlungsform von der Persönlichkeitsstruktur der Patienten abhängt [6].

\section{Physio- und Ergotherapie}

Eine weitere therapeutische Säule, der ein zentraler Stellenwert zukommt, ist durch das aktive und passive Beüben der Patienten im Rahmen der Physio- und Ergotherapie gegeben. Neben den mobilisierenden Übungen sollte auch ein nozizeptives neuromuskuläres Training erfolgen, wobei die Allodynie behandelt wird und die nozizeptiven Afferenzen herunterreguliert werden sollen [15]. Gerade durch das kontinuierliche „Bearbeiten“ der betroffenen Extremität wird das Entstehen eines Neglects - visuelles und funktionelles Ausblenden der erkrankten Extremität - vermieden [8]. Neben der rechtzeitig einsetzenden medikamentösen Schmerztherapie muss bei der Physio- und Ergotherapie der Schwerpunkt auf eine frühzeitige, primär visualisierte und später bewusst empfundene Berührung und Bewegung ohne Schmerz gelegt werden.

Bei der Physiotherapie wird häufig die manuelle Therapie eingesetzt, bei der Traktions-, Friktions- und Kompressionsverfahren angewandt werden. Diese sollen Gelenke mobilisieren und den KapselBand-Apparat aufdehnen [23]. Lymphdränage, Ultraschall- und Laserbehandlungen werden individuell zur Unterstützung der Therapie eingesetzt.

Bei der ergotherapeutischen Behandlung werden alltägliche Belastungsmuster geübt („activity of daily life“: ADL) [8]. Hierunter sollen die Patienten schneller wieder in ihre alltäglichen Arbeiten und in ihr altes Leben eingegliedert werden. Weiterhin werden individuell verschiedene Schienen angepasst, die sowohl dynamisch als auch statisch auf die Verbesserung der Bewegungsumfänge einwirken sollen.

\section{Stationäres (berufsgenossenschaft- liches) Heilverfahren}

Ihm kommt unter allen genannten Behandlungsmaßnahmen bei schweren Fällen eine zentrale Bedeutung zu [16]. 


\section{Chirurgie}

Operative Maßnahmen sind grundsätzlich zurückhaltend einzusetzen. Besonders bei den sympathikusinduzierten Verläufen kann jedoch eine operative Sympathektomie zur Linderung beitragen. Bevor ein solcher Eingriff erfolgt, sollte der Effekt durch eine invasive Sympathikusblockade geprüft werden [19]. In seltenen Fällen können Tenotomien (Kontrakturen) oder auch Arthrodesen sinnvoll sein. Diese Eingriffe sind jedoch bei akuten Verlaufsformen streng kontraindiziert.

Umstellungsosteotomien aufgrund fehlverheilter Schmerz auslösender Frakturen sind nach Möglichkeit außerhalb des akuten Stadiums und nach Ausschöpfung der konservativen Maßnahmen einer CRPSTherapie streng indiziert. Eine frühe Operation im akuten Stadium aufgrund starker Schmerzen infolge Fehlstellung muss indikatorisch immer sehr kritisch geprüft werden.

\section{Weitere Behandlungsmöglichkeiten}

Die in der Vergangenheit häufig empfohlene Gabe von Kalzitonin hat sich in der Klinik langfristig nur als wenig wirksam herausgestellt, sodass hierzu nicht mehr geraten wird [9].

Unter Berücksichtigung der zentralen Komponente bei der Entwicklung und Aufrechterhaltung des CRPS werden neuerdings immer häufiger zentralwirksame Medikamente eingesetzt. Hierzu ist auch Gabapentin zu rechnen, wobei es noch $\mathrm{zu}$ keiner breiten klinische Anwendung gekommen ist [7]. In unserer Klinik konnten wir eine Verbesserung der klinischen Gesamtsituation der Patienten und eine signifikante Reduktion der vorher pathologisch erhöhten Blutflussaktivität in den schmerzassoziierten Arealen auf der Hirnrinde im Rahmen einer Studie mit dem NMDA-Rezeptor-Antagonisten Memantin beobachten [22]. Auch hierzu ist jedoch kritisch anzumerken, dass die Erfahrungen mit einem größeren Patientenkollektiv bislang fehlen.
Trauma Berufskrankh 2006 · 8[Suppl 3]:S329-S332

DOI 10.1007/s10039-005-1066-y

c) Springer Medizin Verlag 2005

H.-E. Schaller $\cdot$ N. Sinis

\section{Behandlung des Morbus Sudeck}

\section{Zusammenfassung}

Die Bezeichnung ${ }_{\text {"complex regional pain }}$ syndrome" (CRPS) beschreibt einen Komplex von Krankheitsbildern, bei denen das erste früher unter dem Namen Morbus Sudeck bekannt war. CRPS gehört in den Formenkreis der neuropathischen Schmerzsyndrome und wird in 3 Typen differenziert. Beim Typ II treten im Gegensatz zum Typ I Beschwerden aufgrund einer Nervenverletzung auf. Typ III beschreibt andere Entitäten wie z. B. die Fibromyalgie. Die genauen Mechanismen, die zum CRPS führen, sind weitgehend unbekannt; in aktuellen Studien wurde eine maßgebliche Beteiligung zentralnervöser Veränderungen auf kortikaler Ebene nachgewiesen. Das CRPS ist durch Schmerzen und trophische Störungen gekennzeichnet. Dazu gehören u. a. Hypertrichose, Ödeme und Hautver-

\section{Treatment of complex regional pain syndrome (formerly known as Sudeck's atrophy)}

\section{Abstract}

Complex regional pain syndrome (CRPS) was formerly known as "Sudeck's atrophy". The disease belongs to the group of neuropathic pain syndromes and is differentiated into three types. Type I is characterized by a lack of nerve lesions, type II by the presence of nerve lesions, and type III by the presence of other entities such as fibromyalgia. The exact pathogenic factors leading to the disease are still unknown and are currently the subject of investigation in various studies. These studies suggest a contribution of the central nervous system to the development and maintenance of CRPS. However, the clinical symptoms are well documented and include pain, autonomic changes and impaired motor function of the affected extremity. Diagnosis is based clinically on signs and symptoms. However, in a few cas- änderungen. Die Diagnose wird überwiegend aufgrund des klinischen Bilds gestellt. Als bildgebende Verfahren werden die Röntgenaufnahme und die Szintigraphie (Veränderungen im Stadium II) empfohlen. Die Therapie sollte stets interdisziplinär erfolgen, wobei die Zusammenarbeit von Chirurgen, Physiotherapeuten, Psychologen und Anästhesisten notwendig ist. Operative Vorgehensweisen sind nur in seltenen Fällen bei nervalen und knöchern bedingten Schmerzen unter strengster Indikationsstellung erforderlich.

\section{Schlüsselwörter}

CRPS · Morbus Sudeck ·

Neuropathisches Schmerzsyndrom .

Trophische Störungen .

Interdisziplinäre Therapie

\begin{abstract}
es radiography and scintiscanning may be useful to finalize the diagnosis. The treatment options are centred on the symptoms of pain, autonomic changes and functional impairment. A multidisciplinary treatment strategy is recommended, with surgeons, anaesthesiologists, physiotherapists and psychotherapists working together. Surgical intervention in this disease is only required in rare cases of neurological and bone pain, and the indications for such intervention are narrow and should be strictly observed.
\end{abstract}

\section{Keywords}

CRPS · Sudeck's disease ·

Neuropathic pain syndrome.

Autonomic changes .

Multidisciplinary therapy 
Tabelle 1

Zusammenfassung der unterschiedlichen Behandlungsstrategien beim CRPS

\begin{tabular}{|c|c|}
\hline Medikamentöse Therapie & $\begin{array}{l}\text { - Nichtsteroidale Antirheumatika } \\
\text { - Opioide } \\
\text { - Trizyklische Antidepressiva } \\
\text { - Gabapentin } \\
\text { - Memantin } \\
\text { - Neurontin }\end{array}$ \\
\hline Psychologische Begleitung & $\begin{array}{l}\text {-Verhaltenstherapie } \\
\text { - Gesprächstherapie }\end{array}$ \\
\hline Interventionelle Therapie & $\begin{array}{l}\text {-Sympathikusblockaden i. v. } \\
\text { - Regionalanästhesien } \\
\text {-Somatische Blockaden }\end{array}$ \\
\hline Physio- und Ergotherapie & $\begin{array}{l}\text { - Manualtherapie } \\
\text { - Neuromuskuläres Training } \\
\text { - Kryotherapie, Ultraschall- und Laseranwendungen } \\
\text { - Lymphdränage } \\
\text {-, activity of daily life“ (ADL) } \\
\text { - Anpassung verschiedener Schienen }\end{array}$ \\
\hline Chirurgische Therapie & $\begin{array}{l}\text {-Sympathektomie } \\
\text {-Arthrodesen } \\
\text { - Tenotomien } \\
\text {-(Korrekturosteotomien) }\end{array}$ \\
\hline
\end{tabular}

\section{Fazit für die Praxis}

Das CRPS gehört in den Formenkreis der neuropathischen Schmerzsyndrome. Es ist gekennzeichnet durch eine prominente Schmerzsituation sowie trophische Störungen wie Hypertrichose, Ödembildung, Glanzhaut usw. Grundsätzlich werden 3 Typen voneinander unterschieden, wobei Typ I im Gegensatz zum CRPS Typ II keine Nervenbeteiligung aufweist. Diagnostisch gilt es v. a., andere Erkrankungen auszuschließen. Dabei steht das klinische Bild bei der Diagnosestellung im Vordergrund.

Die Behandlung des CRPS sollte idealerweise multidisziplinär erfolgen. Hierbei sollten Chirurg, Schmerztherapeut, Psychologe, Physio- und Ergotherapeut eng miteinander zusammenarbeiten. Die Verabreichung zentral wirksamer Medikamente (Gabapentin, Memantin) hat in aktuellen Studien einen positiven Effekt auf den Krankheitsverlauf und die Rekonvaleszenz der betroffenen Extremität belegt. Die Verabreichung dieser Medikamente erfolgt derzeit jedoch weitgehend experimentell. Die langfristigen Ergebnisse an größeren Patientengruppen sind diesbezüglich noch abzuwarten.

\section{Korrespondierender Autor Prof. Dr. H.-E. Schaller}

Klinik für Plastische, Hand-, Rekonstruktive und Verbrennungschirurgie, BG-Unfallklinik, Eberhard-Karls-Universität Tübingen, Schnarrenbergstraße 95, 72076 Tübingen E-Mail: schaller@bgu-tuebingen.de

Interessenkonflikt: Keine Angaben

\section{Literatur}

1. Allen G, Galer BS, Schwartz L (1999) Epidemiology of complex regional pain syndrome: a retrospective chart review of 134 patients. Pain 80: 539-544

2. Birklein F, Handwerker $\mathrm{HO}$ (2001) Complex regional pain syndrome: how to resolve the complexity? Pain 94: 1-6

3. Blumberg H, Jänig W (1994) Clinical manifestations of reflex sympathetic dystrophy and sympathetically maintained pain. In: Wall PD, Melzyck R (eds) Textbook of pain. Churchill Livingstone, Edinburgh, p 691

4. Buchner H, Richrath P, Grunholz J et al. (2000) Differential effects of pain and spatial attention on digit representation in the human primary somatosensory cortex. Neuroreport 11: 1289-1293

5. Ciccone DS, Bandilla EB, Wu W (1997) Psychological dysfunction in patients with reflex sympathetic dystrophy. Pain 71: 323-333

6. Covington EC (1996) Psychological issues in reflex sympathetic dystrophy. In: Jänig W, Stanton-Hicks M (eds) Reflex sympathetic dystrophy: a reappraisal. IASP Press, Seattle, p 195

7. Drummond PD, Skipworth S, Finch PM (1996) Alpha 1-adrenoceptors in normal and hyperalgesic human skin. Clin Sci 91: 73-77
8. Galer BS, Butler S, Jensen MP (1995) Case reports and hypothesis: a neglect-like syndrome may be responsible for the motor disturbance in reflex sympathetic dystrophy (complex regional pain syndrome-1). J Pain Symptom Manage 10: 385-391

9. Gobelet C, Waldburger M, Meier JL (1992) The effect of adding calcitonin to physical treatment on reflex sympathetic dystrophy. Pain 48: 171-175

10. Gulevich SJ, Conwell TD, Lane J et al. (1997) Stress infrared telethermography is useful in the diagnosis of complex regional pain syndrome, type I (formerly reflex sympathetic dystrophy). Clin J Pain 13: 50-59

11. Jänig W, Baron R (2002) Complex regional pain syndrome is a disease of the central nervous system. Clin Auton Res 12: 150-164

12. Maleki J, LeBel AA, Bennett GJ et al. (2000) Patterns of spread in complex regional pain syndrome, type I (reflex sympathetic dystrophy). Pain 88: 259-266

13. Mellik GA, Mellicy LB, Mellnick LB (1995) Gabapentine in the management of reflex sympathetic dystrophy. J Pain Symptom Manage 10: 265-266

14. Merskey H, Bogduk N (1994) Classification of chronic pain: descriptions of chronic pain syndromes and definition of terms, 2nd edn. IASP Press, Seattle, p 115

15. Oerlemans HM, Goris JA, De Boo T et al. (1999) Do physical therapy and occupational therapy reduce the impairment percentage in reflex sympathetic dystrophy? Am J Phys Med Rehabil 8: 533-539

16. Rennekampff H-O, Rabbels J, Pfau M et al. (2003) Die Wertigkeit der BGSW (Berufsgenossenschaftliche stationäre Weiterbehandlung) in der Rehabilitation nach distaler Radiusfraktur. Aktuelle Traumatol 33: 109-114

17. Schott GD (1998) Interrupting the sympathetic outflow in causalgia and reflex sympathetic dystrophy. BMJ 316: 792-793

18. Schwartzman RJ, Kerrigan J (1990) The movement disorder of reflex sympathetic dystrophy. Neurology 40 : 57-61

19. Schwartzman RJ, Liu JE, Smullens SN et al. (1997) Long-term outcome following sympathectomy for complex regional pain syndrome type I (RSD). J Neurol Sci 150: 149-152

20. Sieweke N, Birklein F, Reidl B et al. (1999) Patterns of hyperalgesia in complex regional pain syndrome. Pain 80: 171-177

21. Sindrup SH, Jensen TS (1999) Efficacy of pharmacological treatments of neuropathic pain: an update and effect related to mechanism of drug action. Pain 83: 389-400

22. Sinis N, Haerle M, Gustin S et al. (in press) Memantine for treatment of complex regional pain syndrome of the hand: a preliminary report of six clinical cases. Clin JPain

23. Sinis N, Schaller H-E (2005) CRPS - complex regional pain syndrome. In: Krupp S, Rennekampff HO (Hrsg) Plastische Chirurgie. ecomed, Landsberg, IX-4.3 S 4

24. Stanton-Hicks M (2000) Complex regional pain syndrome (type I, RSD; type II, causalgia): controversies. Clin J Pain 12: 262-269

25. Stanton-Hicks M, Baron R, Boas R et al. (1998) Complex regional pain syndromes: guidelines for therapy. Clin J Pain 14: 160

26. Sudeck $P$ (1931) Die trophische Extremitätenstörung durch periphere (infektiöse und traumatische) Reize. Dtsch Z Chir 234: 596-612

27. Thimineur M, Sood P, Kravitz E et al. (1998) Central nervous system abnormalities in complex regional pain syndrome (CRPS): clinical and quantitative evidence of medullary dysfunction. Clin J Pain 14: 256-267

28. Van de Beek WJ, Schwatzman RJ, Van Nes SI et al. (2002) Diagnostic criteria used in studies of reflex sympathetic dystrophy. Neurology 58: 522-526

29. Van der Laan L, Veldman PH, Goris RJ (1998) Severe complications of reflex sympathetic dystrophy: infection, ulcers, chronic edema, dystonia and myoclonus. Arch Phys Med Rehabil 79: 424-429

30. Zyluk A (1999) The usefulness of quantitative evaluation of three-phase scintigraphy in the diagnosis of post-traumatic reflex sympathetic dystrophy. J Hand Surg [Br] 24: 16-21 